\title{
Managing Traditional Materials in an Online Environment: Some Definitions and Distinctions for a Future Collection Management
}

\author{
Ross Atkinson
}

Collection management (as opposed to collection development) should be understood as the activity of adding value to - or deleting value from $-0 b$ jects of information subsequent to their selection. The management of materials in traditional formats will become increasingly problematic the more we move into an online environment. Although digital resources will sooner or later come to dominate scholarly communication, the effective management of traditional materials will remain essential. While a central goal of libraries must be to manage traditional and digital resources as two aspects of a single service, we must also recognize that all information services will eventually be conditioned by a digital mentality. In order to start planning now for collection management to play a more prominent role in the future of information services, we must begin to define with as much precision as possible the abstract values collection management adds to and deletes from selected information objects.

\section{I} n every age, humankind imagines itself to be moving through a period of transition so acute that the effect borders on the dysfunctional. Our own age is no exception. We long for a simpler past, or for a more focused future, but the fact is that every era is one of profound change, and it is now our turn. Because we have come to understand ourselves mainly in technical terms, we necessarily and correctly view our transition as a consequence of technology, and those of us in academic libraries see our main objective as the transformation of academic information services from a primarily paper-based activity to an increasingly electronic one.

If we had our druthers, we would probably opt to build two libraries-one traditional and one digital; we could then gradually shift resources from the traditional to the digital as needed. Fortunately for academic libraries and higher education, we do not have anything approaching the means that would be required to create such a schism-because if we did, those two libraries would inevitably become politically disaffected and veer apart, and the library as an institution

Ross ATKINSON (RA13@cornell.edu) is Deputy University Librarian at Cornell University, Ithaca, New York. An earlier version of this paper was presented at the 4th Annual University of Minnesota Collection Development Symposium on May 5, 1997. Manuscript received September 3, 1997; accepted for publication November 14, 1997. 
might well under such circumstances become associated primarily with the traditional side. As a result, the library would forfeit much of its political influence, academic information services would be severely impaired, and much time would be lost trying to effect a reconciliation and reunification. Our primary strategy in academic libraries must be, therefore, to bring about this transition through a synthesis of the traditional and the digitaland we will no doubt be obliged to do this using the same amount or less funding than we presently have.

In pursuing this objective, one of the most immediate and obvious challenges we must confront will be the management of traditional materials in a world where information is conveyed increasingly in electronic form. Because we are aiming for synthesis, we must accept that our planning for the place of traditional materials in a digital environment can only be undertaken and understood as a relationship to the role of digital materials in a digital environment-because the traditional and the digital must together form the basis for a single, systematic service. At the same time, we must accept the fact that the more we enter an environment in which most scholarly communication is accomplished in electronic form, the more a digital mentality - conditioned by the qualities of digital sources and methods-will come to dominate and define information services. In the course of this essay, some of the main components of such a mentality will be identified, because it is on the basis of that mentality that we will view and build future services.

In considering dichotomies such as that of the traditional and the digital, we would also do well, at the outset at least, to note what is perhaps the most fundamental dichotomy of information services-that of subject and object. The purpose of information services is and always will be to ensure that local users have access to the right information objects (as defined as those that are needed) within the right time frame. That responsibility can be viewed from either a subjective or an objective perspective. From a subjective position, the goal of information serv- ices is-given a particular set of information objects-to provide local users with the tools and skills they need to make the most effective uses of those objects. But the service can also be approached from the objective perspective: assuming a particular group of local users with clearly defined needs, the goal of the service is to add selected values to specific information objects, such that those objects can be used more effectively to respond to those local needs. While the subjective perspective is most often assumed by reference services, the objective viewpoint is typically that of collection management and development, preservation and cataloging. Much of the political tension in the modern library derives directly and unavoidably from the differences between these two positions. Since my aim in this essay is to approach the problems of the transition from a primarily objective position, we should always bear in mind that a view from the subjective perspective could conceivably lead to different conclusions.

\section{The ObJect}

As befits an approach to information services from the objective direction, let us begin with a description of the current state of the information object. The division between the traditional and digital object is the source (objectively speaking) of the current hybridity of information services. The term "traditional" is now often used for nondigital services (see, e.g., Ewing 1995 and Fecko 1991), so it seems reasonable to extend its use to nondigital objects. It is a conspicuously timedependent term that tends to be used in the midst of a fundamental transition before the vocabulary has caught up; because we do not seem to have an accepted term for nondigital services or objects, we can call them "traditional," by which we mean " of a kind we have worked with until now." The transition will progress much more effectively once we have agreed to replace the term "traditional" with one referring to some quality of the objectrather than one that refers to our present temporal relationship with the object. 


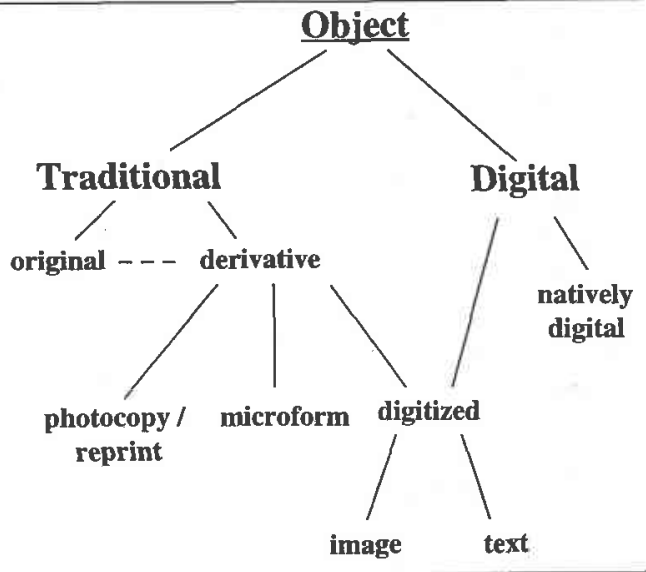

Figure 1. Relationships among Information Objects.

From traditional originals are created traditional derivatives, which include photocopy and microform-but we are now also able to create digital derivatives. Such digital derivatives can be subdivided into the categories of image and text (e.g., ASCII). Digital objects can be divided into the two broad categories of digitized (i.e., derived from a traditional original) and "natively digital"-also a tentative term-meaning materials that have been produced originally in digital form. On the one hand, therefore, we are working with a hierarchy of concepts (vertically in the diagram), but at the same time we perceive a kind of formal or temporal progression-that is, the more one moves horizontally in the diagram from left to right, the further removed one becomes from the concept of the traditional original.

While there are clearly derivatives of digital objects, these are not the same as traditional derivatives. Digital derivatives are more clones than copies, because there is no apparent loss of physical information: digital objects are characterized, therefore, by what we might call derivative parity. In the case of traditional objects, on the other hand, the creation of the derivative necessarily entails some loss of physical content.

We must take care always to distinguish physical from intellectual content.
Physical content is information that is found in the physical construction of the object. Historical bibliography is the study of the history of such physical construction. Analytical bibliography is the study of the relationship of physical and intellectual content. Textual criticism uses historical and analytical bibliography, along with other techniques, to trace the history of the intellectual content of specific texts back to their original archetypes (Greetham 1994). We must wonder what will happen to these disciplines, especially textual criticism, as we move into an increasingly online environment, one of the primary characteristics of which will be derivative parity.

In the traditional environment, we have been willing to accept a certain level of loss of physical content in return for other (usually preservation) advantages. That is the basis for our current traditional derivative decisions. Microforms, photocopies, and reprints eliminate much of the physical content (notably the original format), but retain the typeface; the same is true of digitized images. In the case of the digitized text, on the other hand, all of the physical content is lost, including the typeface, leaving only an encoded symbol string. Both the library preservation profession and the scholarly community remain perhaps still unprepared for the trauma of the loss of the typeface-and there may well even be some resistance to 
designating text-only digitization as a legitimate form of preservation.

Such questions as to how much loss is acceptable stem from a deeply held cultural value in the library, which we might call the warranty syndrome. Librarians in the modern library often seem to assume that one of the most fundamental (objective) responsibilities is to guarantee that an object with which they have been entrusted will remain continuously accessible in all of its parts. This warranty syndrome has certainly contributed to the failure of librarians to achieve effective levels of interinstitutional cooperation in the areas of collection development or remote storage. For one institution to withdraw a title and then depend upon the availability of that title at another institution can require (as a consequence of the warranty syndrome) a level of negotiation and formal interinstitutional commitment that may only be achieved at considerable administrative cost (Hazen 1997). If we insist on guaranteeing access-in other words, eliminating any chances of lossthen it is much more cost-effective to store materials in a local offsite facility than to engage in formal negotiations with other institutions.

The effect of the warranty syndrome on the library's readiness to disregard original physical content (e.g., text-only digitization)-especially with the subsequent withdrawal of the original-remains unclear. What is clear, however, is that the warranty syndrome prohibits the conscious loss of any intellectual content whatsoever. Regardless of whether the loss of all physical content might eventually be acceptable, therefore, the loss of any intellectual content remains generally abhorrent to the traditional library culture. The digital culture, on the other hand, while assuming derivative parity, i.e., no apparent loss of physical content, is rather more habituated to, and accepting of, some loss of intellectual content. The digital culture is characterized by information extracted from remote sites, of which the local user has little knowledge and even less control; it is a culture of Web sites that change every day without warning. Some loss-or "lossiness" as an object attribute-while obviously avoided whenever possible, is nevertheless becoming increasingly understood as part of the price of digital access. This tolerance for some loss of intellectual content conflicts sharply, therefore, with the traditional library culture.

\section{Collection MaNagement OVERVIEW}

Let us now proceed to a simplified overview of the collection development and management process. While the exact definition of collection management remains somewhat obscure, it has often been assumed that collection management should be understood as an expansion of the concept of collection development (see, e.g., Osburn 1990). Collection management then becomes an umbrella term under which collection development is subsumed. However, we need terms that separate policies and actions that drive selection (collection development) from policies and actions that affect the access status of an object subsequent to its selection. The programs and processes by which library materials are selected, therefore, should be termed "collection development," while the process of adding value to objects subsequent to their selection should be considered "collection management." Collection development and management, thus defined, operate on the basis of somewhat different values, and those values must be separated and contrasted in order to be understood and effected.

The activities and concepts of collection development and management should be kept separate also for strategic reasons. There is a chance, perhaps a very good one, that aspects of collection development will not survive the transition to a primarily online environment, because the responsibility for selection in such an environment might be reappropriated by users. When all forms of publication, including monographs, are routinely network accessible, and if an effective level of cataloging can be achieved for those networked resources, then it might well be the user rather than the bibliographer 


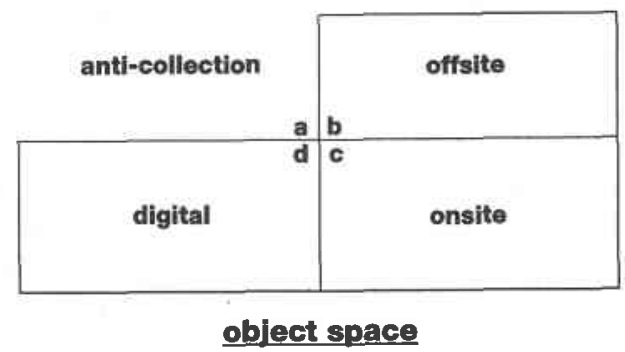

Figure 2. General Model of Objects in a Collection.

who selects material. In that case, some of the knowledge and creativity that have evolved over decades in collection development will need to be transferred to collection management. If, moreover, collection development ceases to be a primary library operation, then the materials budget will no doubt be reclaimed by the institution and somehow apportioned among its users. Should that happen, libraries could lose the funding needed for collection management, if library managers have not previously separated collection development and collection management funding into distinct budgets.

Returning to the present condition, in which the librarian retains responsibility for selection, let us use the term "anti-collection" (Atkinson 1994, 97) for all objects that do not reside in the local collection, or are not made accessible to local users by the library (see figure 2 ). The "anti-collection" is, of course, an abstraction: the set of all objects not in the local collection.

We will assume that offsite and onsite locations (quadrants $\mathrm{b}$ and $\mathrm{c}$ in figure 2) contain objects owned by the library in all traditional formats, including traditional derivatives. In the past thirty years, the great majority of our efforts have been centered in traditional collection development (the movement of objects from a to c). More recently, however, increasing amounts of our attention have shifted to the selection of digital materials (moving natively digital materials from a to d), digitizing materials (i.e., moving information from c to d), or offsite selection (c to b). While most librarians at research libraries have, to be sure, always done some offsite selection, there has been a rapid growth in such selection for remote storage in more recent years--as if all large collections had crossed some kind of line beyond which the transfer of traditional materials offsite has become more politically and bibliographically acceptable. It is as if traditional collections had finally become so large that the central retention of all materials might actually be seen as a potential impediment to access. This increase in the significance of offsite and digital selection calls for a rejuvenation and redefinition of collection management.

Using figure 2, we can then define collection development more exactly as the movement of an information object from the open quadrant (a) into any one of the closed quadrants (b, c, d). Collection management, as we are defining it here, is the movement of an object from any one of the closed quadrants into any other quadrant (including the open quadrant a-that is, weeding).

Pitschmann (1991, 141) has suggested that, in order to facilitate the transition from traditional to digital services, we might consider replacing the term "collection management" with "resource management." On one hand, this suggestion could be problematic because it obscures the significance of object relationships as implied by the term "collection." On the other hand, it is an enticing suggestion because the term "resource" can be applied to both information and economic conditions. The purpose of much of the 


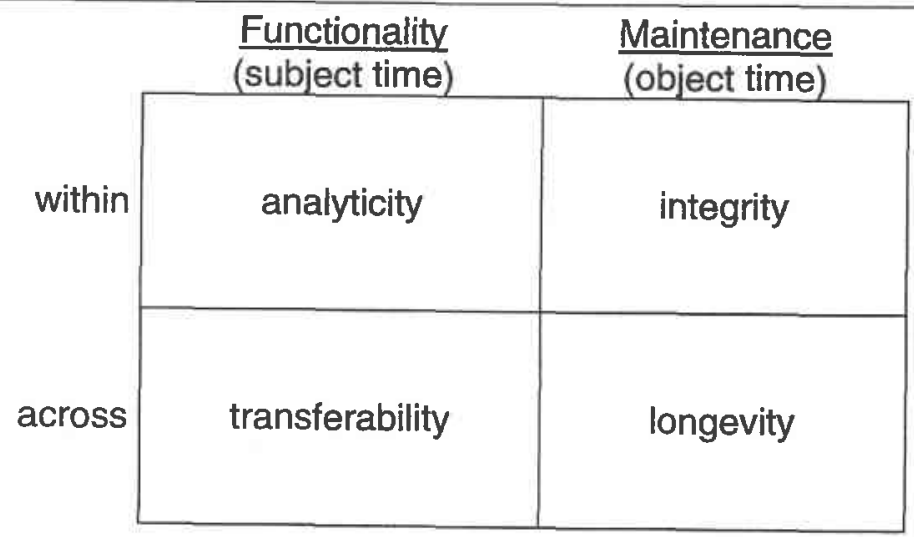

Figure 3. Goal Values.

librarian's activity, after all, is to convert economic resources into (access to) information resources-so that the term "resource management" is a provocative one in that sense. In some cases, moreover, there can also be a conversion of (access to) information resources into (savings of) economic resources.

Collection development is, to be sure, unidirectional: it only converts economic resources into information resources. Collection management also moves in that same direction: it uses economic resources to boost access to information objects that have already been selected. However, one of the most important functions of collection management is that it, unlike collection development, also routinely operates in the opposite directionsaving or increasing economic resources by reducing or eliminating (access to) information resources. Thus, in figure 2 , collection management either increases access at the expense of economic resources (moving objects clockwise, b to c, $\mathrm{c}$ to $\mathrm{d}, \mathrm{b}$ to $\mathrm{d}$ ), or it reduces access, saving economic resources (moving objects counterclockwise, $c$ to $b, b$ to $a, c$ to $a-$ and perhaps eventually $d$ to $c$, or $d$ to a). The criteria for the decisions made by both collection development (in one direction) and collection management (in both directions) are always ultimately based on some application of the prime criterion of potential local utility-i.e., how useful the target object will likely be for the work of current and future local users.

\section{Goal Values}

This description of collection development and management is, needless to say, greatly oversimplified. For one thing, each of the three closed quadrants contains a whole range of service gradations, which are so extensive that it is even possible in some cases to move an object into a higher access quadrant, but actually to reduce its accessibility (as, for example, if one digitized an object-moving it from $\mathrm{c}$ to $\mathrm{d}$-but neglected the index or interface). Rather than relying on the metaphor of object space, therefore, it would be more accurate to define the (objective) work of information services on the basis of the distinguishable values added to information objects. These values added might be divided into two broad categories: (a) functionality-i.e., values that improve the user's ability to manipulate and work within the object, and (b) maintenance-i.e., values ensuring that the object remains stable and available over time (see figure 3).

These two value categories might each be further broken down into two broad goal values (i.e., values, which it is the goal of information services to add to objects). Functionality can be divided into (a) transferability, the capacity to move an 
object from one location to another, and (b) analyticity, the ability to be analyzed, in the sense of breaking down an object into smaller parts for more effective access. Indexing is the main service manifestation of analyticity. In the case of maintenance, the two main goal values would probably be (a) integrity-i.e., ensuring that the content of the object remains stable and uncorrupted as the author intended (what Peter Graham [1994] has called "intellectual preservation"), and (b) longevity, ensuring the object's long-term survival.

The goal values of functionality are concerned with subject-time; they are intended to reduce the amount of time required for the user to gain access to, or to make use of, the object. The goal values of maintenance are concerned with object-time, or the time the object remains extant and intact. The goal values of analyticity and integrity are intended to enhance users' ability to work within an object, while the purposes of transferability and longevity are to move the object across space or time. All of the goal values, therefore, derive from a sense of embedment or context: transferability and longevity entail or presume a larger universe of space and time, just as analyticity and integrity imply the use or quality of the components of an object embedded within its whole.

But despite such conceptual relationships and mutual support among the four goal values, it is nevertheless the case that each of these is operationally and economically distinct; each is independently applicable, so that the addition of one does not entail or require the addition of any other. Because, moreover, the addition (or boosting) of each of these does require an expenditure of economic resources, each is in effect engaged in continuous competition with the others for the library's increasingly scarce economic resources. This competition is the basis (at least from the objective perspective) for the whole economics of information services; all of the library's economic resources (e.g., funding, staff time, staff skill, space, equipment) exist for only one purpose, and that is to add these goal values-individually or in combinationto selected objects. The main criteria for that selection-for deciding which objects should have value added to them, and which values should be added to which objects-will normally be (a) the prime criterion of projected utility, (b) the initial economic value of the object itself, (c) the information philosophy of the library or institution (i.e., an institutional bias for some values over others), and (d) available economic resources.

In making such decisions, the format of the target object is, on the one hand, irrelevant: the librarian should and does aim to add such values to selected objects regardless of format. On the other hand, such decisions will also necessarily be affected by format conduciveness: some formats are more receptive than others for adding or boosting certain values. In general, digital objects are more conducive to functionality. They can be moved across space at nearly the speed of light, and (if in textual form, rather than images) they can be rapidly searched or analyzed. Digital objects are, however, far less conducive (at least at this point in time) to maintenance: they can be easily corrupted, and no standards exist for their long-term archiving and migration (Waters and Garrett 1994, 41). Traditional objects are the opposite of digital objects in this regard: traditional objects are not nearly as conducive to transferability and to analyticity (cataloging and indexing cannot be supplemented with automatic text searching)-but traditional objects are much more conducive (at this time) to maintenance than are digital objects.

\section{The Political Dimension}

If competition among goal values forms the basis for the economics of information services, then we must expect a political superstructure that reflects that competition-and there is indeed a fundamental ideological or political division that is a manifestation of the dichotomy of the two broad value categories, and that is probably as old as the library itself. It is the dichotomy of the information service agency as purveyor on the one hand, and 


\begin{tabular}{cc}
\hline Library & Archive \\
\hline functionality & maintenance \\
subject time & object time \\
short-term & long-term \\
present & past or future \\
synchronic & diachronic \\
flexibility & stability \\
readers & writers \\
sciences & humanities \\
\hline
\end{tabular}

Figure 4. Comparison of Library and Archive Attributes.

as repository on the other. Drawing on the distinction made by Waters and Garrett (1994), we can use the term "library" for the former, and "archive" for the latter. This ideological division is further reflected-and will likely be heightenedby the fundamental dialectic in the digital culture of the search engine and the database (see figure 4 ).

The library side of information services, as opposed to the archive, has vastly greater political power, not only because it is much more associated with the digital culture, but also because its primary user community is present; librarians can rely upon those users to influence current decision-making in the institution. The archival side of information services, on the other hand, views the present in some respects mainly as a conduit through which to move objects from the past into the future. The primary user groups of this side have not yet arrived on the scene, so they have no real political influence. But the archival side has, at the same time, a far greater existential responsibility than the library side, because "life and death" decisions about objects are made; they determine whether objects will survive into the future, while the library side is concerned more about increasing access (reducing access time) to objects that are presently extant. This side increases the availability of objects that are for the most part already available, while the archival side decides whether objects will remain available at all. On the archival side, there is less concern about subject time and more for object time: from the purely archival perspective, it does not really matter how long it will take to gain access to an object, provided that access per se remains possible. From the extreme library side, on the other hand, if an object requires too much time to access, it might as well not exist at all, and any economic resources spent on its maintenance are wasted. (On the value distinctions between librarians and archivists, see McCarthy 1986.)

The library perspective is focused mainly on the needs of readers. The archival perspective is also concerned about reader requirements, but at the same time it represents the writer by ensuring that the object creation remains intact as the writer intended. Also, the library side represents more of a scientific approach, in the sense that it is less concerned about maintaining dated information (which is not to imply that all scientific information necessarily becomes less useful over time), and is well disposed to summary. The archival side, on the other hand, with its concern for history and its focus upon the artifact, might perhaps be more associated with the values of the humanities.

It might sometimes be assumed that the territory of collection development is the library, while collection management is more the concern of the archive. That should not be the case, however, and we must take care not to allow such a political schism to debilitate collection services. Collection management must take into account the needs and goals of both the library and the archive sides-and it should be the main political function and 


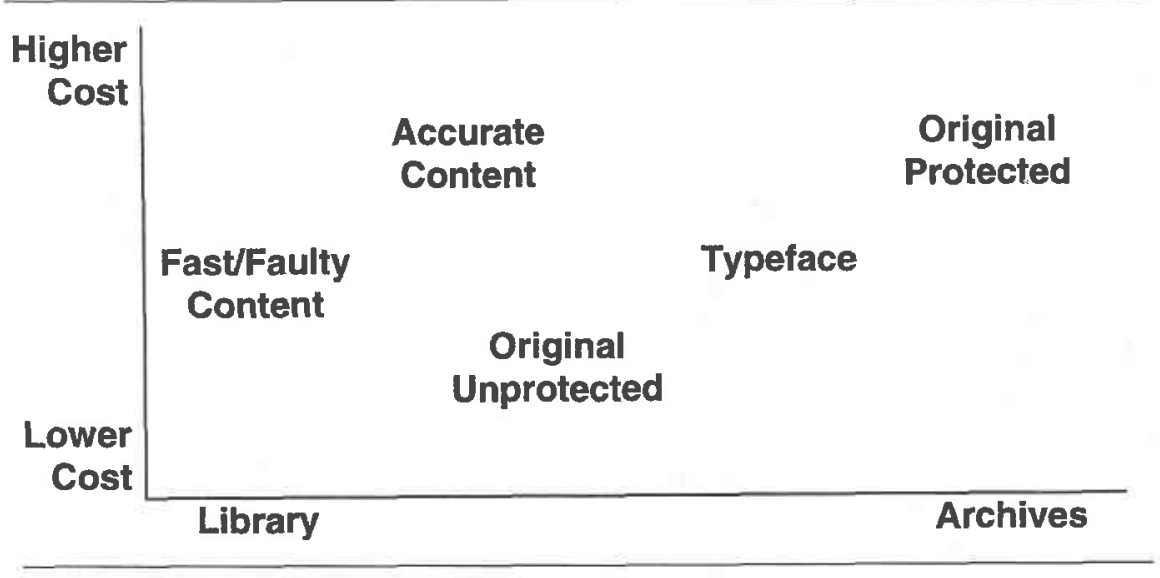

Figure 5. Economic and Political Factors for Providing Access to Objects.

rationale of collection management to connect and synthesize these two fundamentally and historically divergent aspects of library operations.

\section{Traditional Objects}

The options we have for providing access to traditional objects in future will depend upon both economic and political factors (see figure 5). From the archival perspective, maintaining the original object in a protected form (as in a special collection) is the ideal. Under such conditions, the integrity and longevity of both the physical and intellectual content are assured. The cost of providing objects in their original form with a high level of protection, however, can be significant. (The relative costs for these different operations are only assumptions based on experience; they are not a result of any empirical study.) Less preferable from the archival perspective, but presumably somewhat less expensive, is to maintain a reformatted version only, in which the typeface is retained. Because of the reduced costs and the more ready accessibility, this is also a much preferable solution from the library perspective-and even more so, if the reformatting is digital. Less preferable from the archival position, but probably even less expensive, is the maintenance of the original in unprotected form, e.g., offsite; seen from the archival position, this is a deferral of necessary action, but it does mean at least that all physical content is maintained for the moment. From the library position, the unprotected original is somewhat more accessible than some forms of typefaceonly reformatting (notably microform), although it is obviously far less preferable than digitization.

Much more acceptable from the library viewpoint would be a total digitization of the text only, which would render the entire document keyword searchable. This could be, of course, a very expensive undertaking, since unevaluated Optical Character Recognition (OCR) would not provide full accuracy; such an undertaking would require very costly quality control. From the archival standpoint, such a step is even less preferable because it entails a loss of all physical content-but at least the symbol string of the original, representing the intellectual content, is kept intact. Totally unacceptable from the archival side would be fast but not entirely accurate digitization (uncorrected or "dirty" OCR), for this would inevitably entail some loss of intellectual content. From the library perspective, such rapid digitization would be-in some cases-an ideal solution, because it would provide access to (pound for pound) more information at a much reduced cost. (Needless to say, however, in those instances requiring totally accurate content, even the li- 


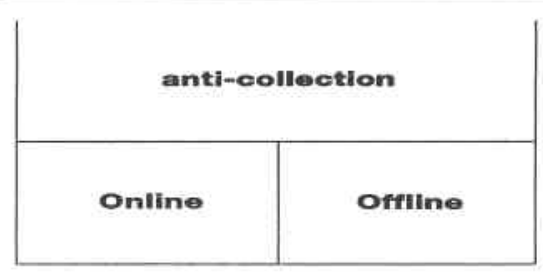

Figure 6. Modified Model for Objects in Collections.

brary position would support fully accurate digitization.)

For more important (potentially higher use) traditional objects, combinations of one option from the library side and one from the archival side are the preferable action. The combination of fast but inaccurate OCR with typeface digitization, for example, is an especially effective option, because it allows the user to search the OCR, but view an image of the typeface; both processes can be done relatively automatically, as opposed to fully accurate OCR, which entails much expensive human intervention. (The current JSTOR project, for instance, uses both OCR and images; the OCR done for JSTOR, however, is subject to quality control, and the project feels it has achieved 99.95\% accuracy as a result. See http:// www.jstor.org/about/production.html.)

Well after much of the action in academic scholarship has shifted online, institutions will doubtless remain custodians of large quantities of paper materials-unprotected originals. The reason for this will be partially economic (it costs less, we assume, to put such originals someplace on a shelf, rather than to convert them). However, the maintenance of unprotected originals will also have political motivations: the unprotected original is a compromise between the library and the archival value directions, and as such will be acceptable to the broadest range of libraries and users.

\section{The Future Model}

As we noted earlier, the movement of objects from the anti-collection to the onsite library has been the primary and dominant focus of academic information serv- ices for centuries. As we move more into a digital environment, however, we must now expect the centrality of the onsite library for information services gradually to dissolve. The onsite collection, (quadrant $\mathrm{c}$ in figure 2), will and should eventually disappear as an independent conceptual entity, and its basic functions will be divided and drawn into the other two quadrants, $b$ (offsite) and d (digital). This does not mean that the onsite library will cease to exist, but rather only that users (and information service providers) will probably come gradually to view any library that is not desktop accessible as being a remote storage facility, even if it is on a central campus. The offsite storage facility is merely more remote than the onsite facility, while traditional objects maintained in collections at other institutions are simply more remote still.

The three closed quadrants-onsite, offsite, digital-in our general model (figure 2), therefore, will ultimately contract into two: online and offline (see figure 6). Given the significance of transferability in the functional value system, we should begin to define objects in the online collection as those that are network accessible at the local institution-that is, not objects that have only the capacity to be network accessible, but rather those that are locally network accessible at the present time.

It is this dialectic of online and offline that should become the operative dichotomy, ultimately replacing the transitional dichotomy of traditional and digital. It is not that the concepts of traditional and digital objects should lose their relevance, but rather that they should be subsumed within this operative dichotomy. While online object space contains only digital objects, offline object space should be seen as holding both digital and traditional objects. Digital objects that are maintained offline are those that are not network accessible, such as stand-alone CD-ROMs. There are also different gradations of offline (and online) access; the more offline the object is, the less accessible it becomes as an information resource-but also, in general, the lower the cost in economic terms of its maintenance 
and functionality.

We can expect three broad categories of objects to reside offline:

1. The most obvious (and possibly largest) category will be objects of low potential use. These will consist mainly of large collections of unprotected traditional objects, although we must assume some digital materials will also fall into this category. Such digital objects will be kept offline because they do not promise high potential utility; this category will include some objects that were once online, but that have now been moved offline for storage purposes, because their local utility has declined.

2. While there will be some objects kept offline because they are considered less important, there will be a second category of objects kept offline because they are more important. These will include all of the protected traditional originals (although some of these might have been digitized as well); most of these will presumably reside in special collections. We must also expect to create and maintain offline backup copies of particularly important digital objects for archival or historical purposes.

3. A third category of materials maintained offline will be those that are either technically unsuitable for online access as well as, more importantly, those for which there are legal impediments to digitization. We do well to remember that libraries do not own the intellectual content of many of the objects they maintainbut rather only, so to speak, the physical content of the copies they have purchased. The owners of the intellectual content-individual publishers-presently have the right and responsibility to decide whether such objects may be digitized for online access, and we must expect that a certain amount of material that is legitimately needed online for scholarly purposes will be kept offline by publishers to protect their investments. If the academy continues to outsource its publishing to (especially commercial) publishers, then it is possible that access to such information will be increasingly restricted, the more we move online. Re-appropriating at least some of the responsibility for specialized scholarly publishing must therefore be one of the highest priorities for the academic community.

\section{The Role of Collection MANAGEMENT}

What are the responsibilities of collection management in an evolving digital environment? To answer this question, we must return to the fundamental values added (depicted in figure 3), bearing especially in mind the factor of format conduciveness. We should recognize that the acts of selection and acquisition add to the selected object all four goal valuestransferability, analyticity, integrity, longevity - to a limited degree; we could, in fact, define selection and acquisition as the modest addition of these values to particular objects. If the object is considered to be especially important, collection management can then boost access to the object by increasing one or some combination of those values. This can be done by increasing further those values to which the object by virtue of its format is already conducive: digital objects can be made more transferable or analyzable, or the integrity and longevity of traditional objects can be further improved. Altematively, or in addition, collection management can move to compensate for values that are less prevalent because of format: the integrity or longevity of digital objects can be boosted by, for example, creating offline back-up copies, or the transferability and analyticity of traditional objects can be increased through digitization.

If the object is determined to be of less importance, the collection management decision may be simply to do nothing, i.e., to leave in place the values added by selection, but not to boost those values in any way. On the other hand, if an object is determined to be of less potential utility to local users than at the time of its selec- 
tion, then collection management can move in the opposite direction, reducing access in order to increase or maintain economic resources. In those cases, for example, digital objects might be moved offline, or traditional objects could be digitized, and their originals discarded. In many (but certainly not all) cases, such adding or subtracting of value - the primary work of collection managementwill be increasingly accomplished by moving objects online and offline.

\section{The Digital Mentality}

We noted at the outset that as the work of scholarship becomes increasingly available in digital form, our thinking about information services in general will be informed by a digital mentality that is conditioned by the special qualities and capabilities of digital objects. We have already drawn some conclusions about that mentality. We have seen, for example, that the old tension in the library culture between the library and the archival value directions is likely to be heightened in future by the fundamental division in the digital mentality between the search engine and the database. We have also speculated that the operative dichotomy for information services (online or offline) should be based upon network accessibility, because transferability appears to be a primary (perhaps dominant) value of functionality, which is more conducive to digital objects. We have also remarked in passing on two other aspects of the digital mentality that are likely to have wide ranging effects on the transformation of information services-loss tolerance and embedment. Let us conclude by considering these attributes more carefully.

As both the library community and its users become increasingly accustomed to some forms of information loss in some circumstances (not only of physical but also intellectual content), we must expect the iron grip of the warranty syndrome to loosen: librarians will no longer be inclined to see as an essential function the provision of total access forever to every object for which they assume responsibility. We can perhaps begin to develop levels of responsibility or warranty con- nected to or derived from the standard collection levels (cf. Bryant 1987 for a definition of the collection levels). This change in culture and philosophy will have a number of important implications. It will mean, for one thing, that we should be able to put in place much more effective programs of cooperative collection management, which will become increasingly necessary for unprotected originals. If we can avoid the high levels of negotiation and item tracking that have sometimes appeared as necessary prerequisites for cooperation in the past, then we can begin to rely more regularly on each other-with the understanding that such reliance will necessarily entail a certain amount of loss. The dissolution of the warranty syndrome should also increase our willingness and ability to make macrodecisions. Such macro-decisions will become more necessary as we are compelled to move more materials offsite. As that work can rarely be done on an item by item basis, the ability to make broad decisions on large groups of objects is essential, but these decisions will unavoidably engender some loss of information for the local user community. The greater tolerance for lossiness, and the concomitant reduction of the warranty syndrome, should also mean that we will be prepared to engage in much more fast-but-less-accurate conversion, e.g. uncorrected OCR. If the choice is between digitizing ten items very accurately and digitizing several hundred items less accurately, we should have the option of choosing the latter in some cases.

We may also conclude that there is indeed an important future for textual criticism in an increasingly online environment. Because of such contrivances as uncorrected OCR, we are perhaps entering an era in which we will see a real renaissance of textual criticism. For economic reasons, librarians will create poor digital copies of objects-and then we will inevitably lose the originals in some instances. If a later age then decides those objects were important, much highly specialized scholarly work will be needed to reconstruct the archetypes of those objects.

The decline of the warranty syndrome 
should also contribute to the final elimination of the myth of comprehensiveness. To collect comprehensively on a subject, or to provide comprehensive access, has always been a highly questionable concept (see Exon and Punch 1997). The fact is that all research and bibliographic searching, no matter how systematic and sophisticated, is necessarily a form of browsing — but it has taken Web browsers to remind scholars (and even some librarians) of that reality. We need, therefore, not only to condone browsing, but to search for ways to improve it. Because of offsite storage, as well as the increasing use of more streamlined cataloging methods, there has probably never been a time in the history of modern libraries when main stacks browsing has been more difficult and unproductive. We need to replace what was lost in the stacks with a new ability to browse online - albeit with the understanding that online browsing is something very different from traditional browsing (Heim 1993, 24-27).

Equally important for the future of information services will be the heightened sensibility for embedment. Both transferability and analyticity entail embedment. Transferability is the potential to move objects within a wider universe, while analyticity is the capacity for the user to move within the object. Thus while every database is an object, we must also recognize that every object is a database. We should anticipate, therefore, a loosening or broadening of the concept of the object. Presently we feel that we have a firm grasp in the traditional environment on the nature or definition of the object. We think of it as a document. It is a book or a journal-but we know an object is also a chapter of a book, or an article within a journal. But does that mean that several articles in the same journal might also conceivably together constitute an object? Would it be possible to think of several journals on the same subject area as an object? How about all of the publications of a single author? The separate publications of a group of associated authors? A number of items in different formats on the same subject areas? A collection of items in the same place? A collection of items in different places that are administratively linked? A collection of items in the same geographic region?

It is by asking such questions that we begin perhaps to gain some insight into the novelty and complexity of the collection management environment we are now entering. It is a highly volatile environment, in which information services will be called upon as never before to balance the library and the archival sides, the capacity for reformulation and the prerequisite for stability. New objects can and will be created increasingly from previously extant objects, but the traces and components of those extant objects will need also to be safeguarded in some cases. It will be neither loss tolerance nor embedment that paves the way to the new information environment, but rather their dynamic combination - because each implies the other.

The digital mentality, with its heightened sensitivity to embedment, will cause us to realize more than ever that the collection is itself an information object. Collection development has always had the potential to be viewed and practiced as a form of authorship; because, if every text is to some extent a compilation of previous texts, then the collection is a kind of textand the building of the collection is a kind of authorship. Opportunities and requirements for that same creativity will now be found through collection management, as we move increasingly online, and as technology provides us with abilities for text manipulation and object definition. Even if the responsibility for selection is partially or totally transferred to users in the online environment, so that collection development ceases to be a key responsibility of the academic librarian, the creative skills and knowledge of collection management-the ability to change the relationships of objects to each other, and of users to objects, by adding values to (or deleting values from) objects already selected-will remain a fundamental information service.

\section{Works Cited}

Atkinson, Ross. 1994. Access, ownership, and the future of collection development. In Collection management and development: 
issues in an electronic era, ed. Peggy Johnson and Bonnie MacEwan: 92-109. Chicago: American Library Association.

Bryant, Bonita, ed. 1989. Guide for written collection policy statements. Collection management and development guides, No. 3. Chicago: American Library Association.

Ewing, M. Keith and Robert Hauptmann. 1995. Is traditional reference service obsolete? Journal of academic librarianship 21: 3-6.

Exon, F.C.A. and Keith F. Punch. 1997. The self-sufficient library collection: A test of assumptions. Journal of the American Society for Information Science 48: 11-16.

Fecko, Mary Beth and Linda Langschied. 1991. The impact of electronic journals on traditional library services. The serials $l i$ brarian 21: 185-87.

Graham, Peter S. 1994. Intellectual preservation: Electronic preservation of the third kind. Washington, D.C.: Commission on Preservation and Access.

Greetham, D.C. 1994. Textual scholarship: An introduction. New York: Garland.

Hazen, Dan C. 1997. Cooperative collection development: Compelling theory, inconse- quential results? In Collection management for the 21st century: A handbook for librarians, ed. G.E. Gorman and Ruth H. Miller: 263-83. Westport, Conn.: Greenwood.

Heim, Michael. 1993. The metaphysics of virtual reality. New York: Oxford Univ. Press.

McCarthy, Paul H. 1986. Archives under library administration: Points of convergence and conflict. Journal of library administration 7: 17-34.

Osburn, Charles B. 1990. Collection development and management. In Academic libraries: Research perspectives, ed. Mary Jo Lynch: 1-37. Chicago: American Library Association.

Pitschmann, Louis A. 1991. Organization and staffing. In Collection management: A new treatise, ed. Charles B. Osburn and Ross Atkinson: 125-43. Greenwich, Conn. JAI Press.

Waters, Donald and John Garrett. 1994. Preserving digital information: Report of the Task Force on Archiving Digital Information. Washington, D.C.: Commission on Preservation and Access. 